\title{
ON SOME INEQUALITIES FOR UNITARILY INVARIANT NORMS
}

\section{XiaOhui Fu And ChuAnJiang He}

Abstract. In this paper, we present several inequalities for unitarily invariant norms by using the convexity of the function $g(r)=\left\|A^{r} X B^{2-r}+A^{2-r} X B^{r}\right\|$ on the interval $[0,2]$. Our results are refinements of some existing inequalities.

Mathematics subject classification (2010): 47A63.

Keywords and phrases: Unitarily invariant norm, convex function, inequality.

\section{REFERENCES}

[1] R. Bhatia, Matrix Analysis, Springer-Verlag, New York (1997).

[2] R. BhatiA, C. DAVIS, More matrix forms of the arithmetic-geometric mean inequality, SIAM J Matrix Anal Appl. 14, 132-136 (1993).

[3] X. ZHAN, Inequalities for unitarily invariant norms, SIAM J Matrix Anal Appl. 20, 466-470 (1998).

[4] R. Bhatia, F. KitTANeH, Notes on matrix arithmetic-geometric mean inequalities, Linear Algebra Appl. 308, 203-211 (2000).

[5] S. WAng, L. Zou AND Y. JiAng, Some inrqualities for unitarily invariant norms of matrices, J Inequal Appl. 2011, 10 (2011).

[6] L. Zou, C. HE, On some inequalities for unitarily invariant norms and singular values, Linear Algebra Appl. 436, 3354-3361 (2012).

[7] P. S. Bullen, A Dictionary of Inequalities, Pitman Monogarphs and Surveys in Pure and Applied Mathematics, vol. 97. Longman, Harlow (1998).

[8] F. Kittaneh, On the convexity of the Heinz means, Integr Equ Oper Theory. 68, 519-527 (2010). 